\title{
Lock-free GaussSieve for Linear Speedups in Parallel High Performance SVP Calculation
}

\author{
Artur Mariano \\ Institute for Scientific Computing \\ Technische Universität Darmstadt \\ Darmstadt, Germany \\ artur.mariano@sc.tu-darmstadt.de
}

\author{
Shahar Timnat \\ Department of Computer Science \\ Technion - Israel Institute of Technology \\ Haifa, Israel \\ stimnat@cs.technion.ac.il
}

\author{
Christian Bischof \\ Institute for Scientific Computing \\ Technische Universität Darmstadt \\ Darmstadt, Germany \\ christian.bischof@tu-darmstadt.de
}

\begin{abstract}
Lattice-based cryptography became a hot-topic in the past years because it seems to be quantum immune, i.e., resistant to attacks operated with quantum computers. The security of lattice-based cryptosystems is determined by the hardness of certain lattice problems, such as the Shortest Vector Problem (SVP). Thus, it is of prime importance to study how efficiently SVP-solvers can be implemented.

This paper presents a parallel shared-memory implementation of the GaussSieve algorithm, a well known SVP-solver. Our implementation achieves almost linear and linear speedups with up to 64 cores, depending on the tested scenario, and delivers better sequential performance than any other disclosed GaussSieve implementation. In this paper, we show that it is possible to implement a highly scalable version of GaussSieve on multi-core CPU-chips. The key features of our implementation are a lock-free singly linked list, and hand-tuned, vectorized code. Additionally, we propose an algorithmic optimization that leads to faster convergence.
\end{abstract}

Keywords GaussSieve, SVP, parallel, mul1ti-core CPU, lock-free

\section{Introduction}

Cryptography is mainly used to protect information that is sent over an insecure channel. In 1996, Ajtai found out that some lattice problems have interesting properties for cryptography, such as averagecase hardness [1]. Cryptosystems based on lattices are said to fall within the realm of lattice-based cryptography, a rapidly expanding field since Ajtai's discoveries on lattice problems. Lattices are discrete subgroups of the $m$-dimensional Euclidean space $\mathbb{R}^{m}$, with a strong periodicity property. A lattice $\mathcal{L}$ generated by a basis $\mathbf{B}$, a set of linearly independent vectors $\mathbf{b}_{1}, \ldots, \mathbf{b}_{n}$ in $\mathbb{R}^{m}$, is denoted by:

$$
\mathcal{L}(\mathbf{B})=\left\{\mathbf{x} \in \mathbb{R}^{m}: \mathbf{x}=\sum_{i=1}^{n} \mathbf{u}_{i} \mathbf{b}_{i}, \mathbf{u} \in \mathbb{Z}^{n}\right\}
$$

where $n$ is the rank of the lattice. When $n=m$, the lattice is said to be of full rank. When $m$ is at least 2, each lattice has infinitely many different bases.
Lattice-based cryptography is particularly attractive since latticebased cryptosystems are believed to be quantum immune, i.e., resistant to attacks operated with quantum computers. Lattice-based cryptosystems can only be broken when specific lattice problems can be solved in a timely manner. As the security of these systems is estimated based on the performance of the algorithms that solve their underlying problems, highly optimized and parallelized solvers are needed to realistic estimations. One of the underlying problems in lattice-based crypto-systems is to find the shortest vector in a given lattice, a problem referred to as the Shortest Vector Problem (SVP). The SVP consists in finding the nonzero vector $\mathbf{v}$ of a given lattice $\mathcal{L}$, whose norm $\|\mathbf{v}\|$ is the smallest among the norms of all nonzero vectors in the lattice $\mathcal{L}$ and is denoted by $\lambda_{1}(\mathcal{L})$. The problem can be stated for every norm; In this paper, we address the Euclidean norm, the most common in this context. An algorithm that solves the SVP is called a SVP-solver.

SVP-solvers work faster on reduced lattice bases, i.e. lattice bases with short, nearly orthogonal vectors. The main algorithms that can be used to reduce lattices are the Lenstra-Lenstra-Lovász (LLL) and the Block Korkine Zolotarev (BKZ) algorithms (cf. [8]). LLL sparked a new era of research on lattice basis reduction. Lattice basis reduction algorithms can be used to solve approximate solutions of the SVP. In fact, for lattices in two dimensions, the LLL algorithm solves the SVP exactly. Lattice basis reduction algorithms are widely used in many fields and in cryptanalysis, including in different types of cryptography, such as knapsack cryptosystems and special settings of RSA. In this paper, we use BKZ to pre-reduce the lattices in which we solve the SVP on.

There are three main classes of SVP-solvers: sieving algorithms, enumeration algorithms and algorithms based on the Voronoi cell of a lattice (see [8] for a comprehensive overview). Sieving algorithms were introduced in 2001, via the AKS algorithm [2], and extended in 2010, with algorithms that improve the complexity of AKS [11]. An asymptotic better variant of the AKS algorithm, called ListSieve, as well as its efficient heuristic GaussSieve, were presented by Micciancio et al. [11]. While ListSieve was considered of low practicability, the authors did also present an efficient heuristic of ListSieve, called GaussSieve. A theoretical improvement of ListSieve was presented by Pujol et al. [16]. Recently, a three-level sieving heuristic, with better time and space complexity than ListSieve, was proposed [18]. However, it is still unclear if it can perform better than GaussSieve, because neither there is a practical implementation of it nor is the time complexity of GaussSieve known.

To this day, two parallel versions of GaussSieve were proposed, either with limited scalability $[10,12]$, or requiring some parameter whose optimal value cannot be calculated upfront [6]. The implementation proposed in [6] requires the number of samples used per 
iteration to be given as input. This is a major problem because the optimal value for this parameter is not known upfront, and suboptimal values increase the time that the algorithm takes to converge. In Section 3.2 we overview both implementations in detail.

At this point in time, the fastest probabilistic approach to solve the SVP in practice, in terms of running time, seems to be enumeration solvers with extreme pruning. However, sieving algorithms are still interesting because (1) they are asymptotically better than enumeration $\left(2^{\mathcal{O}(n)}\right.$ vs. $\left.2^{\mathcal{O}(n \log n)}\right)$, (2) they can take more advantage of specific lattices, such as ideal lattices, than enumeration algorithms (see [15], Section 6.1) and (3) some advances in sieving algorithms have been published during the last years and it is expected that further optimizations will be proposed in the next years. For instance, this paper proposes one algorithmic optimization that enables GaussSieve to converge faster.

Our contribution is three-fold. In addition to the aforementioned algorithmic optimization of the GaussSieve algorithm, we present a parallel, scalable multi-core implementation that slightly relaxes the properties of GaussSieve, with negligible impact on the time for convergence. Our implementation delivers high levels of performance due to hand-tuned and vectorized code. Finally, we propose an extension to the lock-free list proposed in [5], which is used as the core of our implementation.

This paper is organized as follows. Section 2.1 provides relevant notation and recaps some definitions, and Section 2.3 explains the GaussSieve algorithm. In Section 3, we overview related work, both concerning SVP-solvers in general, and parallel implementations of the GaussSieve algorithm in particular. Section 4 explains our approach in detail and the implemented optimizations. Section 5 shows how our implementation performs and compares with other parallel implementations of GaussSieve. Finally, Section 6 concludes the paper and provides some future lines of research.

\section{Preliminaries}

\subsection{Notation and definitions}

The Euclidean norm of a vector is the distance spanned from the origin of the lattice to the point given by the vector $\mathbf{v}$, i.e. $\|\mathbf{v}\|=\sqrt{\sum_{i=1}^{n} \mathbf{v}_{i}^{2}}$, where $\mathbf{v}_{i}$ is the $\mathrm{i}^{t h}$ coordinate of $\mathbf{v}$. We use the term zero vector for vectors whose norm is zero, i.e., the origin of the lattice. Vectors and matrices are written in bold face, vectors are written in lower-case, and matrices in upper-case, as in vector $\mathbf{v}$ and matrix $\mathbf{M}$. The dot product of two vectors $\mathbf{v}$ and $\mathbf{p}$ is denoted by $\langle\mathbf{v}, \mathbf{p}\rangle$. The lattice $\mathcal{L}$ generated by a basis $\mathbf{B}$ is denoted by $\mathcal{L}(\mathbf{B})$. We now give two important definitions in the context of the GaussSieve algorithm:

Definition 1 - Gauss-Reduced: Two vectors $\mathbf{p}$ and $\mathbf{v} \in \mathcal{L}(\mathbf{B})$ are said to be Gauss-reduced (with respect to each other) when $\min (\|\mathbf{p} \pm \mathbf{v}\|) \geq \max (\|\mathbf{p}\|,\|\mathbf{v}\|)$ holds true. A simple routine to reduce two vectors $\mathbf{p}$ and $\mathbf{v}$ was presented in [11], and is referred to as the Reduce kernel. When this routine is invoked bi-directionally, i.e., Reduce (p,v) and Reduce (v,p), $\mathbf{p}$ and $\mathbf{v}$ become Gauss-reduced.

Definition 2 - Pairwise-reduced: A set or a list $L$ of vectors is pairwise-reduced, i.e., all its elements are pairwise-reduced, when every pair (p,v) $\forall \mathbf{p}, \mathbf{v} \in L$, is Gauss-reduced.

We also recall the definitions of speedup $S_{p}$ and efficiency $E_{p}$, presented in Equation 2. and 3, respectively.

$$
S_{p}=\frac{T_{1}}{T_{p}},
$$

where $T_{p}$ is the program's execution time with $p$ processors.

$$
E_{p}=\frac{S_{p}}{p}=\frac{T_{1}}{p T_{p}}
$$

\subsection{The Shortest Vector Problem}

Virtually every lattice problem has to do with distances. In particular, the SVP consists in finding the non-zero vector $\mathbf{v}$ of a given lattice $\mathcal{L}$, whose norm $\|\mathbf{v}\|$ is the smallest among the norms of all nonzero vectors in the lattice $\mathcal{L}$. This norm is usually denoted by $\lambda_{1}(\mathcal{L})$ or simply $\lambda_{1}$, if it is clear what lattice is concerned. As a result, the SVP can formally be defined as the computation of a vector $\mathbf{v} \in \mathcal{L}(\mathbf{B}) \backslash\{0\}$ where $\|\mathbf{v}\|=\lambda_{1}(\mathcal{L}(\mathbf{B}))$. The problem can be stated for every norm; In this paper, we address the Euclidean norm, the most common in this context.

The picture of the best SVP-solvers has been changing during the last decade. In particular, enumeration and sieving have been two concurrent approaches, competing for the position of the best SVP-solver. Sieving algorithms were first thought to be impractical, until the AKS algorithm was proven to be practical, in 2008 [13], even though still uncompetitive with enumeration routines. In 2010, Micciancio et al. presented GaussSieve, the first sieving heuristic that outperformed enumeration routines [11]. However, in the same year, Gama et al. proposed the extreme pruning approach for enumeration algorithms [4], which drove GaussSieve, and consequently sieving algorithms, out of the podium. This approach reduces the probability of enumeration algorithms to find the shortest vector of a lattice, but it reduces their running time by a much higher factor. The method shuffles the basis and runs the extreme pruned enumeration on it, repeating the process until the shortest vector is found. In practice, enumeration with extreme pruning becomes probabilistic and a probabilistic stopping criterion, as in sieving algorithms, must be used instead.

Although enumeration algorithms became the main line of research in lattice-based cryptanalysis, several studies on sieving algorithms were still published since 2010 [6, 9, 12, 14, 15, 17, 18]. As mentioned before, sieving algorithms are still of prime importance, because they can be adapted to take advantage of special lattice structures, in contrast to enumeration algorithms [15]. Sieving algorithms might have attracted less attention than enumeration algorithms also because they have been thought to be difficult to parallelize. In particular, Fitzpatrick et al. presented several improvements to GaussSieve, which offer considerable speedups in practice [3]. From those, we highlight an approach that enables to estimate the angle between two vectors, which can be implemented very efficiently with vectorized routines. Mariano et al. also showed very recently that ListSieve, an algorithm categorized as impractical, is actually practical, especially in massively parallel architectures [10].

In this paper, we show that GaussSieve can, in fact, be parallelized and implemented in a very effective manner, by slightly relaxing its properties. We hope that this might help to shift the attention of the community towards sieving algorithms.

\subsection{The GaussSieve algorithm [11]}

The algorithm is based on sampling lattice vectors and building a list $L$ of shorter (of smaller norm) and shorter vectors. The sampled vectors, referred to as samples, undergo a two-stage reduction process. For each sample $\mathbf{v}$, this process is based on (1) reducing $\mathbf{v}$, when possible, with every vector $\mathbf{p}$ in $L$, thus obtaining $\mathbf{v}^{\prime}$, and (2) reducing every possible vector $\mathbf{p}$ in $L$ with $\mathbf{v}^{\prime}$. As a result, the list $L$ will only hold Gauss-reduced vectors. The algorithm also employs a stack $S$ to keep vectors that are temporarily removed from $L$.

A collision occurs whenever a sample is reduced to the zero vector in stage (1). If this occurs, the stage (2) of the reduction 
process is not executed and the number of collisions is incremented. Otherwise, stage (1) does not generate a zero vector but a v' instead, and stage (2) is executed. In stage (2), the algorithm checks if any vector $\mathbf{l} \in L$ can be reduced against $\mathbf{v}$ '. All such vectors are temporarily removed from $L$, reduced against $\mathbf{v}^{\prime}$ and pushed to the stack $S$. At the beginning of each iteration, the algorithm checks if $S$ contains any vector. If this holds, a vector from $S$ is used as a sample, otherwise a new vector is sampled.

This is iteratively executed until a certain stopping criterion, $K \geq c$, where $K$ is the number of collisions, is met. By then, the shortest vector is expected to be in $L . c$ is usually set in the form $c=$ $\alpha \times \mathrm{mls}+\beta$, where mls is the maximum size of $L$ up to that point. The workflow of the algorithm is shown in Algorithm 1. Although the samples can be generated by any algorithm, they are typically generated with Klein's algorithm, as in [7]. Klein's algorithm has very good theoretical guarantees, and it samples vectors according to a distribution that is statistically close to Gaussian (the variance is arbitrary). This is particularly desirable for GaussSieve, since no direction in space is privileged, and collisions are mostly generated only after the shortest vector is found $[13,14]$.

The algorithm is not trivially parallelizable. At a fine-grained level, while stage (1) of the reduction process is easily parallelizable, phase (2) is not. At a course- grained level, the list $L$ would be read and written by multiple threads, which is not safe unless some sort of synchronization is used. In Section 3.2, we describe the approaches that were followed to parallelize the algorithm. Our approach is based on parallelizing the algorithm at a course-grained level, employing a scalable, thread-safe mechanism that permits the use of $L$ with minimal synchronization.

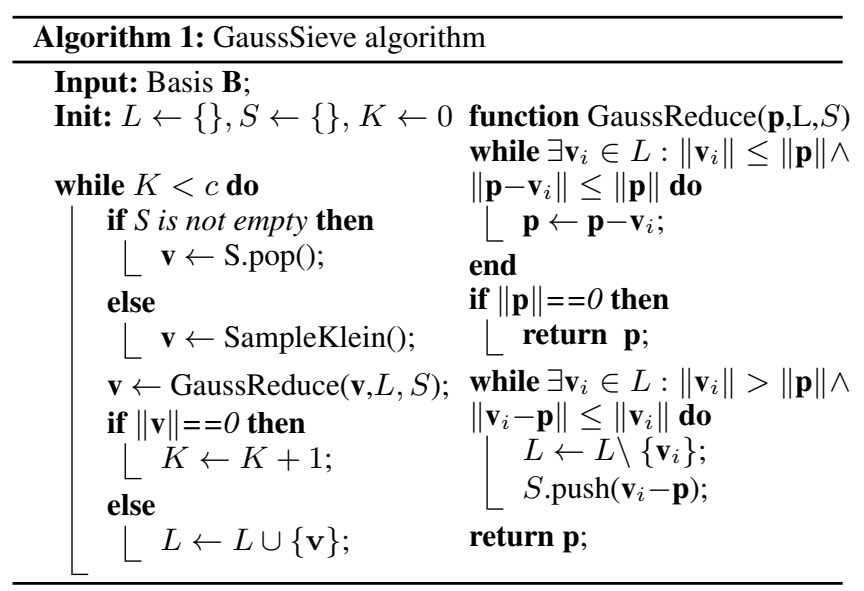

\section{Related Work}

In this section we overview the available implementations of SVPsolvers and the parallel implementations of GaussSieve.

\subsection{Sequential SVP-solvers}

There are various implementations of SVP-solvers. The fplll ${ }^{1}$ library implements several algorithms on lattices, mainly relying on floating-point computations. As far as the SVP is concerned, it includes a floating-point implementation of the Kannan-FinckePohst algorithm, here on referred to as fplll's svp. This is currently considered the most efficient sequential available implementation of an enumeration routine without pruning. Panagiotis Voulgaris published a C++ sequential implementation ${ }^{2}$ of GaussSieve, here

\footnotetext{
${ }^{1} \mathrm{http} / / /$ perso.ens-lyon.fr/damien.stehle/fplll/

${ }^{2}$ http://cseweb.ucsd.edu/ pvoulgar/impl.html
}

on referred to as the gsieve library, used in the experiments of the original GaussSieve paper [11]. In this paper, we compare the performance of our sequential implementation with both. To the best of our knowledge, there are no available implementations of enumeration-based routines with extreme pruning.

\subsection{Parallel implementations of GaussSieve}

In 2011, Milde et al. published a first parallel version of the GaussSieve algorithm [12]. The implementation consists of a ring structure connecting several instances of GaussSieve, each containing a local list $L$ and a private stack $S$. Each instance is then executed by a thread, which samples vectors, one by one, reduces them against its local list $L$, and hands them out to the ring structure. Each vector floats around the ring structure, by means of buffers, where it is picked by every thread, and reduced against the local list therein. When the vector returns to the thread that released it, it is added to the local list of that thread.

In the results shown in [12], the implementation does not scale well for more than 4 threads (the efficiency is $\geq 85 \%$ only for 4 or fewer threads), because the number of iterations required for convergence increases with the number of threads. This happens because the local lists progressively hold more and more vectors that are not Gauss-reduced with all the other vectors in the remaining lists: if any vector $\mathbf{v}$ of a thread $i$ passes by the local list of thread $t$, between the release of a vector $\mathbf{p}$, by thread $t$, and its commitment to the local list of thread $t, \mathbf{v}$ and $\mathbf{p}$ will never be reduced against each other. The bigger the number of threads running on the system, the more often this case occurs and therefore the greater the number of iterations required for convergence.

In 2013, Ishiguro et al. proposed another parallel version of GaussSieve [6], for shared and distributed memory systems, with better scalability on shared memory systems ${ }^{3}$, at least up to 8 threads. Their implementation is based on the following property of union of pairwise-reduced sets: if two sets $A$ and $B$ are pairwisereduced and every pair of vectors $(\mathbf{a}, \mathbf{b})$ is Gauss-reduced, $\forall \mathbf{a} \in$ $A, \mathbf{b} \in B$, then $A \cup B$ is also pairwise-reduced.

The algorithm sets up a list $V$ with $r$ samples, with $r$ provided as input, and applies a 3-stage reduction process. In stage (1), the sample vectors in $V$ are reduced against the vectors in $L$, identically to the original GaussSieve algorithm, but in parallel. Every vector that is modified in this process is added to the stack $S$, otherwise it is moved to a $V$ ' list, whose elements can not be further reduced with any element in $L$. In stage (2), the original vectors in $V^{\prime}$ are reduced against one another, in parallel. We emphasize that the reduced vectors are the original vectors in $V$, otherwise this would represent a dependency. As a result, the vectors in $V^{\prime}$ must be copied to a separate variable before the reduction against other vectors. The modified vectors are moved onto the stack $S$, whereas the unmodified vectors are moved to a list $V$ ”. In stage (3), each thread reduces a part of $L$ against the elements in $V$ ". Again, if any vector is changed in the reduction process, it is added to the stack $S$, otherwise it is added to $L^{\prime}$. Like $V^{\prime \prime}, L^{\prime}$ is also pairwise-reduced.

Once this 3 -stage process is concluded, $L^{\prime}$ and $V^{\prime \prime}$ are merged to create the new list $L$ and the list $V$ is filled up with the vectors that are in $S$ (if they do not total $r$ vectors, more are generated and added to $V$ ). This whole process re-starts until the number of collisions $K$ reaches a certain threshold $c$. However, if $K$ reaches $c$ in the midst of one iteration, that whole iteration, which contains $r$ samples, is still fully executed. The original algorithm, on the contrary, stops as soon as the number of samples reaches the desired boundary $c$.

This approach has two major drawbacks. While it exposes parallelism and permits good scalability on shared memory systems, (1) the use of $r$ samples increases the computation that is necessary

\footnotetext{
${ }^{3}$ In this paper, the results on distributed memory will not be addressed.
} 
for convergence and (2) the optimal value of $r$ is never known upfront. In fact, there is a close relation between how optimal $r$ is and the runtime of the algorithm (see Figure 3(a) in [6]). Additionally, the optimal value for this parameter varies, very likely, from lattice to lattice and from dimension to dimension. Therefore, $r$ must be chosen on the basis of empirical tests, but there is no point in solving the SVP on the same lattice twice. We can therefore assume that a non-optimal parameter will always be chosen in first place.

There are also some implementation details that are not discussed in the paper, and it is unclear how they are solved and how much overhead they cause in the implementation. For example, in the three stages of the reduction process, several vectors are moved to the stack $S$, which represents a dependency. In Algorithm 4, in the Appendix of [6], only three kernels, which exclude the insertions in $S$, are run in parallel. This means that that insertions in $S$ are sequential, which limits scalability.

Our implementation attains much better scalability figures than in [12] and better performance than the results reported in [6], whose code is undisclosed, thereby preventing us from carrying out thorough comparisons.

\section{Lock-free GaussSieve Implementation}

The root of the main problems in the implementations described in Section 3.2 is the distribution of the original list L. In [12], vectors fluctuate between a number of different data-structures and might fail at encounter one another during the reduction process. In contrast to the previously described implementations, we keep the vectors in a central list $L$, safely accessible by every thread concurrently. Unlike scenarios with multiple local lists, as in [12], vectors are likely to see one another during the reduction process, because they are physically close to one another. In particular, not only two vectors $\mathbf{v}$ and $\mathbf{p}$ are likely to encounter each other during the reduction process, but reduced versions of these vectors are also likely to encounter one another, as discussed in Section 4.2. This approach is also better than [6], because (1) it does not need extra parameters for which the optimal values are not known upfront and (2) it stops as soon as the threshold of collisions is reached.

Our implementation is written in $\mathrm{C}++$, uses OpenMP to manage a team of threads and uses gsieve's implementation of Klein's. It sets up a shared lock-free list $L$, which is an enhanced version of Harris's linked list [5]. Each thread executes the original workflow of the algorithm: they sample a vector $\mathbf{v}$, reduce it against every vector $\mathbf{p}$ in $L$, obtaining $\mathbf{v}^{\prime}$, and reduce every vector $\mathbf{p}$ in $L$ against $\mathbf{v}^{\prime}$. Each thread has also a private stack $S$, were $\mathbf{p}$ ' $=\operatorname{Reduce}\left(\mathbf{p}, \mathbf{v}^{\prime}\right)$ is moved onto, whenever $\mathbf{p}$ ' $\neq \mathbf{p}$. Our lock-free implementation relies on the compare-and-swap atomic primitive for synchronization.

\subsection{Enhanced lock-free list}

We implemented the lock-free linked list described in [5], with some modifications and extensions. Each node in the list represents a vector, and includes an array data $[\mathrm{N}]$, which represents the coordinates of the vectors, a long norm, which holds the norm of the vectors, and a pointer Node $*$ next to the next element in the list. $\mathrm{N}$ is the dimension of the lattice.

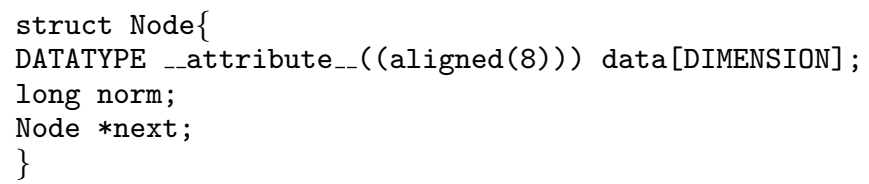

data is an array of either ints or shorts. The list is ordered by increasing norm, similarly to key in [5]. For the atomics, we used compiler built-in functions.
Vectors in the shared list should not be directly modified, since if two threads concurrently modify the same vector the result could be erroneous. If a thread wishes to modify a vector, it should instead remove it from the list and insert a modified version of it. This requires a slight change in the Reduce function of the gsieve library. This function tests if $\mathbf{p}$ should be reduced against $\mathbf{v}$, changing $\mathbf{p}$ if the test holds true, removing it from $L$ afterwards. We split Reduce into two other functions, testReduce and eReduce, thus ensuring that vectors are never modified while in $L$.

testReduce tests if $\mathbf{p}$ should be reduced against $\mathbf{v}$. It does so by computing dot $=\langle\mathbf{p}, \mathbf{v}\rangle$ and then testing whether $(a b s(2 * \operatorname{dot}) \leq$ $\|\mathbf{v}\|)$. If the result is true, then $\mathbf{v}$ is removed from $L$, and copied to a different variable. This copy of $\mathbf{v}$ is updated using eReduce, and pushed onto the stack $\mathbf{S}$ (the private stack of the relevant thread). If the result is false, eReduce is not called. To avoid performance losses, the variable dot is passed by reference to testReduce, so it can be reused in eReduce without any recalculation.

As for stage (1) in the GaussSieve function, a sample vector $\mathbf{p}$ is reduced against all the vectors $\mathbf{l} \in L$ such that $\|\mathbf{l}\| \geq\|\mathbf{p}\|$. This is a straightforward process, because all the threads can read the list concurrently (as mentioned, elements are never written while in $L$ ), and therefore reduce their own samples. After this process, $\mathbf{p}$ is to be inserted in $L$, such that $L$ remains ordered.

Both the insert and remove methods in Harris's linked list use an internal search method, which searches for a given key (a vector norm in our case) from the beginning of the list. However, in the lock-free GaussSieve implementation, it is superfluous to search for the desired norm from the beginning of $L$. If during the traversal a vector $\mathbf{p}$ that should be reduced against $\mathbf{v}$ is found and consequently needs to be removed from $L$, the location of $\mathbf{p}$ is known at the time. Similarly, if during the traversal a vector bigger than $\mathbf{v}$ is found, and $\mathbf{v}$ should be inserted right before it, the location for the insertion is known. We extended Harris's linked list to support insertions and removals without traversing the list from scratch. However, it is important to note that the known locations cannot be used blindly, since other threads may change the list concurrently.

The original search method in Harris's linked list returns two nodes: the first node in the list with a key at least as large as the given search key, and its predecessor. A successful insert operation inserts the new node between these two returned nodes. A successful remove operation removes the second of these nodes (which contains the desired key).

We extended Harris's linked list with two new methods, insertViaPointer, and removeViaPointer. These methods receive an extra parameter, searchPointer, which is ideally the designated predecessor of the new node (for an insert) or the predecessor of the node to be removed (for a remove). These methods are similar to the original insert and remove methods, but call a modified version of the search method, which receives the searchPointer parameter as well.

The modified search method, searchFromMiddle, begins the search from the given searchPointer, instead of from the beginning of the list. Ideally, this parameter points to the first node (of the pair of nodes to be returned), and the search will be completed after very few steps. The searchFromMiddle method also helps if several new nodes were concurrently inserted to the list immediately after the searchPointer, and one of them is now the wanted predecessor, since starting the search from the searchPointer is still much preferable to starting it from the beginning. Moreover, thanks to special traits in Harris's linked list, the searchPointer can potentially be helpful even if the node it points to has already been deleted. Harris's linked list is designed in such a way that allows the traversal to continue through deleted nodes. Such nodes have a special mark that marks them as logically deleted before they are physically removed from the list. 
If during the traversal of the nodes that starts from the searchPointer, searchFromMiddle finds a node that is both (1) with a norm smaller than the desired norm and (2) not deleted, then there is no need to start the search from the beginning of the list. Often, the searchPointer itself points to such a node, the desired one for our purposes. If searching from the middle does not find such a node, then there is no choice but to revert back to searching from the beginning, such as in Harris's original search method.

This approach is optimistic. In most cases, the given pointer to the insertViaPointer or removeViaPointer is the immediate predecessor, and searchFromMiddle will be completed at once. Even if this is not the case, searchFromMiddle still has a good chance of saving a considerable amount of time by avoiding a search from the beginning of the list. In a small number of cases, due to concurrency, the only choice is to search from the beginning.

Note that in terms of functionality, insertViaPointer and removeViaPointer are identical to the regular insert and remove, but they have the potential of saving considerable time.

\subsection{Relaxation of GaussSieve properties}

The implementation proposed by Milde et al., in [12], relaxes the properties of GaussSieve in the sense that several pairs of vectors might never be Gauss-reduced during the execution of the algorithm. Let us consider a scenario with 2 threads, where a given vector $\mathbf{v}$ and a given vector $\mathbf{p}$ are released, at the same time, by threads 1 and 2, respectively. If the vector $\mathbf{p}$ is reduced against the vectors in the local list of thread 1 before $\mathbf{v}$ is in that list, $\mathbf{p}$ and $\mathbf{v}$ will never be reduced against each other (missed reduction). $\mathbf{v}$ and $\mathbf{p}$ will eventually be added to the local lists of threads 1 and 2, respectively. Each vector will possibly fluctuate between the local list of the thread that released it and the private stack of that same thread, but $\mathbf{v}$ and $\mathbf{p}$ will never be reduced against each other.

Similarly to Milde et al. [12], we relax the properties of the GaussSieve heuristic, although to a much smaller degree. In our implementation, it is possible that a given vector $\mathbf{p}$ is reduced against the elements in the lock-free list $L$, while another vector $\mathbf{v}$ is already in the system but not in the list $L$. For instance, $\mathbf{v}$ may lie on the private stack $S$ or under the reduction stage (1) of another thread. If this occurs, $\mathbf{p}$ will not be reduced against $\mathbf{v}$, but it is possible that $\mathbf{v}$ is reduced against $\mathbf{p}$. This will be verified if, when $\mathbf{v}$ is later on reduced against all the elements in $L, \mathbf{p}$ remains unchanged and still in $L$. In fact, this is likely to happen, because if $\mathbf{v}$ lies on the stack of one thread, it means that it will soon be reduced against all the elements in $L$. Assuming this scenario, where $\mathbf{v}$ changes to $\mathbf{v}^{\prime}$, it is also possible that a reduced version of $\mathbf{p}, \mathbf{p}$ ', is later on reduced against $\mathbf{v}^{\prime}$. In fact, this is very likely to happen, because every vector fluctuates between the private stack $S$ of each thread and the lock-free list $L$. When the element is picked from the private stack of one thread, it is reduced against all the elements in $L$. In a nutshell, while it is possible that a vector $\mathbf{p}$ is not reduced against another vector $\mathbf{v}$ when it should be, it is likely that reduced versions of these vectors are eventually reduced against one another, unlike Milde et al..

Although this is a different behaviour from the original algorithm, its impact on the convergence speed is minimal, otherwise the scalability of our parallel version would be considerably affected, as in [12]. As the number of missed reductions grows with the number of threads in our approach, it might happen that a very conservative stopping criterion has to be used for a large number of threads. However, the output of our implementation was, for all our experiments (up to 64 threads), identical to the sequential version.

\subsection{Code optimizations}

The dominant kernel of the implementation is the calculation of the dot product $\langle\mathbf{p}, \mathbf{v}\rangle$, whose result is used to determine if a vector $\mathbf{p}$ should be reduced against a vector $\mathbf{v}$. We have vectorized this kernel for vectors with both integer and short entries, using 128-bit registers from SSE 4.2 (4 integers or 8 shorts are packed per register). While integer entries did not result in overflow during our experiments, short entries did. To overcome this, we used the instruction PMADDWD, which multiplies point-wise short entries, producing temporary signed, doubleword results. The adjacent doubleword results are then summed up and stored in the destination operand, thus keeping overflow losses. We show performance results pertaining to the vectorization of this kernel in Section 5.2.1.

Another relevant optimization, that improved our implementation in up to $15 \%$, is to reduce the number of (dynamic) memory allocations of vectors. As we developed our own module for stack $S$, we save one memory allocation when removing a vector from a list and inserting on the stack $S$. As mentioned, this is first copied to a different variable, allocated within the GaussReduce function, which is then used as a stack element.

\subsection{Algorithmic optimizations}

Similarly to enumeration algorithms, that have been optimized with techniques such as extreme pruning, sieving algorithms can also be modified to converge faster. We observed that GaussSieve converges in fewer iterations when:

- (opt1) the samples used during the sieving process are short;

- (opt2) the reduction of the samples is primarily done against vectors that are short themselves.

From here on, these cases will be referred to as opt 1 and opt2, respectively. In order to attain opt1, we changed parameter $d$, in Klein's algorithm, to $\log (n) / 70$, thus forcing it to sample shorter vectors. This was addressed in [6] first hand (see Section 5.4). It is known that the shorter the samples in sieving algorithms, the faster the algorithms converges. Although our experiments confirmed the performance gains reported in [6], we noticed that, with this modification, the sampler can become a very heavy or even the dominant kernel within the algorithm. Moreover, with this optimization, the default stopping criterion becomes insufficient for lattices in dimensions up to 60 , a problem that was not addressed in [6].

opt 2 can be achieved by ordering the reduction of sampled vectors differently than in the gsieve library. According to the description of the algorithm, in [11], the reduction of a sampled vector abides by the following condition:

$$
\begin{aligned}
\text { while }\left(\exists \mathbf{v}_{i} \in L:\left\|\mathbf{v}_{i}\right\| \leq\|\mathbf{p}\| \wedge\left\|\mathbf{p}-\mathbf{v}_{i}\right\|\right. & \leq\|\mathbf{p}\|) \text { do } \\
& \mathbf{p} \leftarrow \mathbf{p}-\mathbf{v}_{i}
\end{aligned}
$$

In the gsieve library, the possible reduction of a sample $\mathbf{p}$ is tested against every element in $L$, and the process restarts from the beginning of the list only (1) after testing the reduction of p against every element in $L$ and (2) if at least one reduction is successful. Our implementation, on the other hand, restarts the process from the beginning of the list whenever a reduction is successful, therefore forcing the algorithm to use the shorter vectors in $L$ in first place. Despite of this difference, both implementations abide by Equation 4, but our reduction process is more efficient.

\section{Results}

We divide this section into three subsections. In Section 5.1, we compare the performance of our implementation, running with one thread, with the best disclosed sequential implementations of some SVP-solvers. In Section 5.2, we present results concerning the vectorization and the scalability of our implementation, as well as the impact of lattice reduction on GaussSieve. Finally, in Section 5.3, we compare our implementation with the results of the previous parallel implementations of GaussSieve [6, 12]. 
The analysis was carried out with several random lattices, generated with Goldstein-Mayer bases, in multiple dimensions, available on the SVP-challenge ${ }^{4}$ website. All lattices were generated with seed 0 . Table 1 provides the specifications of the two test platforms, with 16 and 64 cores. The 16-core machine runs Ubuntu 11.10, kernel 3.0.0-32-generic, whereas the 64-core machine runs SUSE Linux Enterprise Server 11 SP3, kernel 3.0.101-0.29-default. The results were obtained on the 16-core machine, except in Section 5.2.3, where we present the scalability tests for both machines.

\begin{tabular}{l|c|c} 
& 16-core machine & 64-core machine \\
\hline \#Sockets & 2 & 8 \\
CPU manufacturer & Intel & Intel \\
Model number & E5-2670 & E7-8837 \\
Launch date & Q1'12 & Q2'11 \\
Micro-architecture & Sandy Bridge & Nehalem-C \\
Frequency & $2600 \mathrm{MHz}$ & $2667 \mathrm{MHz}$ \\
Cores & 8 & 8 \\
SMT & Hyper-threading & Not available \\
L1 Cache & $8 \times 32 \mathrm{kB}$ iC+dC & $8 \times 32 \mathrm{kB}$ iC+dC \\
L2 Cache & $8 \times 256 \mathrm{kB}$ & $8 \times 256 \mathrm{kB}$ \\
L3 Cache & $20 \mathrm{MB}$ shared & $24 \mathrm{MB}$ shared \\
System memory & $128 \mathrm{GBs}$ & $1 \mathrm{~TB}$ \\
\hline
\end{tabular}

Table 1. Specifications of the test platforms. SMT stands for Simultaneous multi-threading, $\mathrm{iC} / \mathrm{dC}$ for instruction/data cache.

The code was compiled with Intel icpc 13.1.3, but the experiments in Section 5.2.1 include results for GNU $\mathrm{g}^{++} 4.6 .1$ as well. We used the -02 optimization flag on both compilers, since it was slightly better than -03 . Every experiment was repeated three times and the best sample was chosen, although the runtimes usually were quite stable among different runs. The elapsed time of lattice reduction is not included in the results. Target norms (cf. definition in Section 5.3.2) were never used, except in Section 5.3.2.

\subsection{Performance comparison in sequential}

In this section, we compare our parallel lock-free implementation of GaussSieve, from here on referred to as plfgsieve, running with a single thread, with (1) the fplll's svp call and (2) the gsieve library, both overviewed in Section 3.1. The codes were compiled with icpc and the bases were BKZ-reduced. We used NTL's implementation of $\mathrm{BKZ}^{5}$, with block-size 20 . Although $\mathrm{fplll}$ has itself an implementation of BKZ, we stuck to NTL's implementation of BKZ for all of the three SVP-solvers, since different implementations of BKZ impact the performance of the solvers.

With higher block-sizes, BKZ finds the shortest vector per se, thus making comparisons of the solvers impossible: the execution time of the SVP-solvers would be nearly zero, since the elapsed time of the lattice reduction process is not included in the measurements.

We deactivated optimization opt1 described in Section 4.4 in our implementation, because it renders the algorithm unstable for lattices in dimensions lower than 60, as also mentioned in Section 4.4. As for the stopping criterion, described in Section 2.3, we set $\alpha=0.1$ and $\beta=200$, for both gsieve and plfgsieve.

As Figure 1 shows, our version outperforms clearly the gsieve library, due to the use of vectorization and opt2. In particular, the difference of performance grows with the dimension of the lattice. For instance, our implementation is $\approx 2.56 \mathrm{x}$ faster for a lattice in dimension 50 , but $\approx 4.45 \mathrm{x}$ and $\approx 5.61 \mathrm{x}$ faster for lattices in dimensions 66 and 68. Moreover, the runtime of GaussSieve increases with the dimension of the lattice, regardless of the implementation.

\footnotetext{
$\overline{{ }^{4} \mathrm{http}: / / \text { www.latticechallenge.org/svp-challenge/ }}$

${ }^{5} \mathrm{http}: / /$ www.shoup.net/ntl/
}

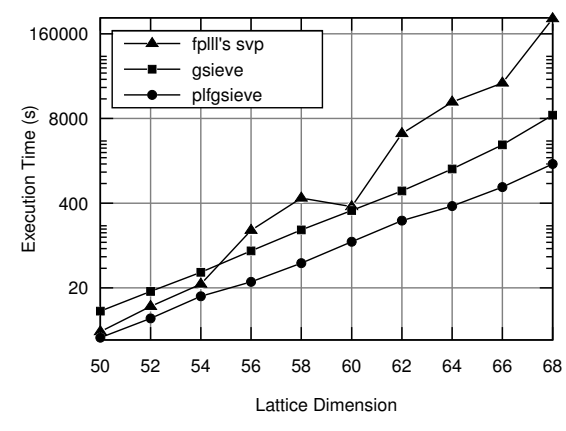

Figure 1. Execution time, in seconds, for fplll's svp, gsieve and plfgsieve ( 1 thread), on lattices in dimensions 50-68 (less is better).

The running time of fplll's svp, on the contrary, does not necessarily increase on a lattice in a higher dimension. For instance, the fplll's svp is faster on the lattice in dimension 60 than on the lattice in dimension 58. The fplll's svp is slower than both implementations of GaussSieve for lattices in higher dimensions than 54. In particular, the differences become very significant for high dimensions (e.g. $\approx 40 \mathrm{x}$ slower than plfgsieve for dimensions 64 and 66 ).

\subsection{Performance of parallel lock-free GaussSieve}

This section shows the assessment of the vectorization of the dot product kernel on our implementation, the impact of lattice reduction on GaussSieve, and its scalability.

\subsubsection{Vectorization and compiler's impact}

This section shows a quantitative performance evaluation of the vectorization of the kernel that computes the dot product $\langle\mathbf{v}, \mathbf{p}\rangle$, the dominant kernel of the proposed implementation. The kernel was isolated and ran on synthetic vectors, in dimension 80 , both 8 - and 16-bytes aligned. In order to obtain solid numbers, the kernel was run 100 million times and the average performance was calculated.

Table 2 presents the results of the benchmarks, when the data array, introduced in the beginning of Section 4.1, is 8-byte aligned. It includes the number of Cycles Per Element (CPE), where an element is a multiplication of $\mathbf{v}_{i}$ and $\mathbf{p}_{i}$, in the dot product $\langle\mathbf{v}, \mathbf{p}\rangle$. The results differ considerably for different data-types and between hand- and compiler-vectorized code. Both compilers perform equally on code that is not hand-vectorized, for short arrays, whereas $g^{++} 4.6 .1$ performs better than icpc 13.1 .3 for not hand-vectorized code on int arrays, by a factor of $\approx 1.39 x$. This picture changes for hand-vectorized code: icpc 13.1.3 performs better than $\mathrm{g}^{++} 4.6 .1$ for integer arrays, by a factor of $\approx 2.74 \mathrm{x}$, and a factor of $\approx 2.70 \mathrm{x}$ is gained in operations on short arrays.

For memory that is 16-byte aligned, the difference between the performance of both compilers is very similar to the results with 8-byte aligned memory. With integers, the performance of all scenarios and both compilers is actually, for two decimal places, the same as with memory that is 8-byte aligned. When it comes to short arrays, icpc 13.1.3 performs worse in code that is not hand-vectorized, but maintains the very same levels of performance in hand-vectorized code. gcc 4.6.1, on the other hand, performs worse in both hand and not hand-vectorized code. These results are shown in Table 3.

A thorough comparison between the compilers is beyond the scope of this paper. The results in this section assess the vectorization of the dot product kernel that we devised, and the selection of the best compiler for our implementation, running on the machine 


\begin{tabular}{cc|c|c|c} 
& \multicolumn{2}{c}{ icpc 13.1.3 } & \multicolumn{2}{c}{ g++ 4.6.1 } \\
\cline { 2 - 5 } & Time (s) & CPEs & Time (s) & CPEs \\
\cline { 2 - 4 } & \multicolumn{2}{c}{ Not hand-vectorized } \\
\hline integers & 9.618 & 3.126 & 6.900 & 2.242 \\
shorts & 7.012 & 2.279 & 7.000 & 2.274 \\
\hline \multicolumn{5}{c}{ Hand-vectorized } \\
\hline integers & 0.698 & 0.227 & 1.910 & 0.621 \\
shorts & 0.364 & 0.118 & 0.982 & 0.320 \\
\hline
\end{tabular}

Table 2. Runtime, in seconds, and CPE of the dot product kernel, compiled with both icpc 13.1.3 and g++ 4.6.1. The time concerns 100 million runs of the kernel. Memory is 8-bytes aligned.

selected for benchmarks. On the basis of these results, the results in the remaining sections were obtained with icpc 13.1.3, except when said otherwise, and 8-byte aligned data.

\begin{tabular}{cc|c|c|c} 
& \multicolumn{2}{c|}{ icpc 13.1.3 } & \multicolumn{2}{c}{ g++ 4.6.1 } \\
\cline { 2 - 4 } & Time (s) & CPEs & Time (s) & CPEs \\
\cline { 2 - 4 } & \multicolumn{2}{c}{ Not hand-vectorized } \\
\hline integers & 9.611223 & 3.123647 & 6.906026 & 2.244458 \\
shorts & 7.488067 & 2.433622 & 7.952487 & 2.584558 \\
\hline \multicolumn{4}{c}{ Hand-vectorized } \\
\hline integers & 0.697577 & 0.226713 & 1.911845 & 0.621350 \\
shorts & 0.363973 & 0.118291 & 1.054764 & 0.342798 \\
\hline
\end{tabular}

Table 3. Runtime, in seconds, and CPE of the dot product kernel, compiled with both icpc 13.1.3 and g++ 4.6.1. The time concerns 100 million runs of the kernel. Memory is 16-bytes aligned.

\subsubsection{Impact of lattice reduction}

While it is known that lattice reduction interferes with the performance of GaussSieve, the degree of this interference is not entirely known. In particular, different block-sizes in BKZ might greatly change the performance of GaussSieve. One of the reasons why the optimality of lattice reduction in this context is very hard to estimate, is because it depends not only on the dimension of the lattice but also from lattice to lattice. This means that different parameters of BKZ might be optimal for a certain lattice $\mathcal{L}$ in a given dimension $n$, but might be suboptimal for a different lattice $\mathcal{Q}$, even if $\mathcal{Q}$ is in the same dimension $n$.

Although this subject deserves a thorough analysis by itself, we did conduct a small, yet useful, investigation on this matter. This enables fair comparisons with other implementations of GaussSieve, such as those shown in Section 5.3. In particular, we tested our implementation, running with 32 threads, on a 80-dimensional random lattice BKZ-reduced with different blocksizes, ranging from 26 to 42 . The results are shown in Figure 2.

Considering the execution time of GaussSieve exclusively, the optimal block-size for the lattice reduction process, with BKZ, is 42. However, the execution time of $\mathrm{BKZ}$ increases with the block-size, and becomes a significant portion of the overall elapsed time for block-sizes bigger than 36. This means that even when GaussSieve is executed in a short time-frame, the combined execution time might be substantially higher than in cases where BKZ is run with a small block-size. For instance, GaussSieve executes approximately 8 times faster when BKZ reduces the lattice with block-size 42 instead of 34 . However, the combined elapsed time is almost 2 times smaller when BKZ runs with block-size 34 . This

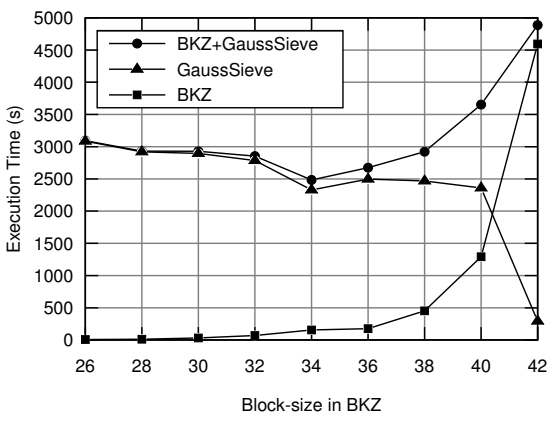

Figure 2. Execution time, in seconds, of GaussSieve, BKZ and both combined for different block-sizes in BKZ.

is interesting because it seems a common practice to omit the execution time of the lattice reduction process, when reporting the execution times for SVP-solvers (e.g. [6, 12]).

The results in the following sections concern lattices that are BKZ-reduced with block-size 32 (except when said otherwise), because it is the most effective value from those in which the lattice reduction process represents $<5 \%$ of the overall elapsed time.

\subsubsection{Scalability}

The scalability of our implementation on the 16-core machine was measured for random lattices in dimensions 60, 70 and 80. Lower dimensions are either solved very quickly or the lattice reduction process finds the shortest vector per se, rendering a scalability analysis worthless. Running the implementation in higher dimensions, on the other hand, is impractical for a single thread.

We conducted two sets of experiments. In the first set, opt2, described in Section 4.4, was not activated. In the second set, on the contrary, opt 2 was activated. BKZ ran with block-size 20 for dimensions 60 and 70, and with block-size 32 for dimension 80. Running the implementation on lattices in dimensions 60 and 70 with the same block-size, of 32 , is particularly fast, and no significant conclusions can be drawn about the results. Moreover, the parameter $d$, in dimension 60 , was $\log (n) / 30$, because the default stopping criterion is insufficient if $d$ is $\log (n) / 70$ instead. The data array, which holds the coordinates of the vectors, was set to hold shorts in both sets of experiments.

Figure 3 shows the execution time for the first set of experiments, for 1-32 threads. The application scales linearly for up to 16 threads. The speedup for 16 threads, in both 60 and 80 dimensions, is almost linear. The use of two sockets (which involves the use of interconnecting CPU buses) does not seem to impair the scalability of our implementation, since it scales linearly for a lattice in dimension 70. The scalability is limited for the lattice in dimension 60, probably due to the small workload that is entailed. For the lattice in dimension 80 , it is possible that the scalability is hurt by the relaxation of the GaussSieve properties on this particular lattice. As shown in Table 4, efficiency levels are very high for the three cases, varying between $83.5 \%$ and $102.25 \%$. In fact, superlinear speedups are achieved for dimension 70 in 2 cases. Moreover, the implementation benefits from SMT (rows are highlighted in light gray in Table 4), since a considerable part of the workflow is memory-bound.

Figure 4 shows the results for the second set of experiments. In dimension 60, linear speedups are achieved for up to 8 threads and an almost linear speedup is achieved for 16 threads. Dimension 70 unveiled a problem that might occur depending on the parametrization of the sampler. opt 1 forces Klein's kernel to output shorter vectors, which renders the kernel heavier and less scalable. The scal- 


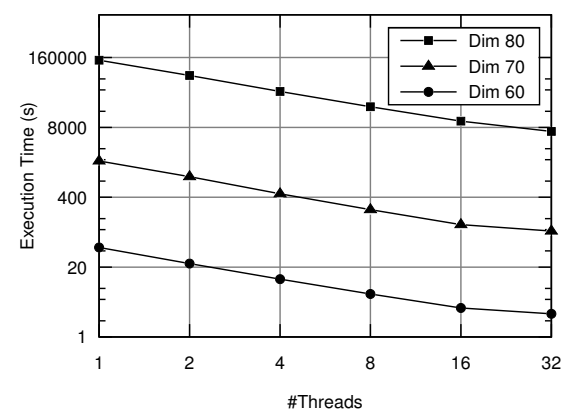

Figure 3. Scalability of our implementation on the 16-core machine (with SMT) for 1-32 threads. Results for lattices in dimensions 60, 70 and 80. BKZ's block-size is 32. opt1 is turned off.

\begin{tabular}{c|c|c|c|c|c|c} 
& \multicolumn{2}{c}{ Dimension 60 } & \multicolumn{2}{c}{ Dimension 70 } & \multicolumn{2}{c}{ Dimension 80 } \\
\hline Threads & $\mathrm{S}$ & $\mathrm{E}$ & $\mathrm{S}$ & $\mathrm{E}$ & $\mathrm{S}$ & $\mathrm{E}$ \\
\hline \multicolumn{8}{c}{ First set of trials } \\
\hline 2 & $2.00 \mathrm{x}$ & $100 \%$ & $1.96 \mathrm{x}$ & $98.00 \%$ & $1.92 \mathrm{x}$ & $96 \%$ \\
4 & $3.88 \mathrm{x}$ & $97.00 \%$ & $4.09 \mathrm{x}$ & $102.25 \%$ & $3.82 \mathrm{x}$ & $95.5 \%$ \\
8 & $7.35 \mathrm{x}$ & $91.88 \%$ & $8.06 \mathrm{x}$ & $100.75 \%$ & $7.35 \mathrm{x}$ & $91.88 \%$ \\
16 & $13.36 \mathrm{x}$ & $83.50 \%$ & $15.36 \mathrm{x}$ & $96.00 \%$ & $13.58 \mathrm{x}$ & $84.88 \%$ \\
32 & $17.18 \mathrm{x}$ & $53.69 \%$ & $20.25 \mathrm{x}$ & $63.28 \%$ & $21.20 \mathrm{x}$ & $66.25 \%$ \\
\hline \multicolumn{7}{c}{ Second set of trials } \\
\hline \multicolumn{8}{c}{} \\
\hline 2 & $1.83 \mathrm{x}$ & $91.85 \%$ & $1.91 \mathrm{x}$ & $95.50 \%$ & $1.93 \mathrm{x}$ & $96.50 \%$ \\
8 & $3.84 \mathrm{x}$ & $96.00 \%$ & $3.48 \mathrm{x}$ & $87.00 \%$ & $3.83 \mathrm{x}$ & $95.75 \%$ \\
16 & $7.34 \mathrm{x}$ & $91.75 \%$ & $4.97 \mathrm{x}$ & $62.13 \%$ & $7.22 \mathrm{x}$ & $90.25 \%$ \\
32 & $13.32 \mathrm{x}$ & $83.25 \%$ & $5.66 \mathrm{x}$ & $35.38 \%$ & $12.64 \mathrm{x}$ & $79.00 \%$ \\
\hline
\end{tabular}

Table 4. Speedups (S) and Efficiency (E) of our implementation running on three random lattices (dimensions 60, 70 and 80). BKZ's block-size set to 32 . SMT is used in grayed out rows.

ability of this kernel is hurt by the use of, among others, a rand()alike function. As this becomes the dominant kernel with this optimization, the scalability of the whole implementation is reduced. In fact, this is the only case where our implementation does not benefit from SMT. This problem is mitigated for higher dimensions, where the sampler is no longer the dominant kernel, as proven by the results in dimension 80 . It is unclear if higher dimensions might benefit from even more strict parameters in Klein's algorithm, which

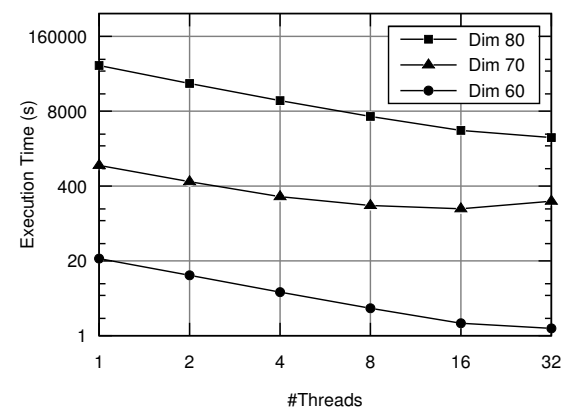

Figure 4. Scalability of our implementation on the 16-core machine (with SMT) for 1-32 threads. Results for lattices in dimensions 60,70 and 80 . BKZ's block-size is 32 . opt1 is turned on.

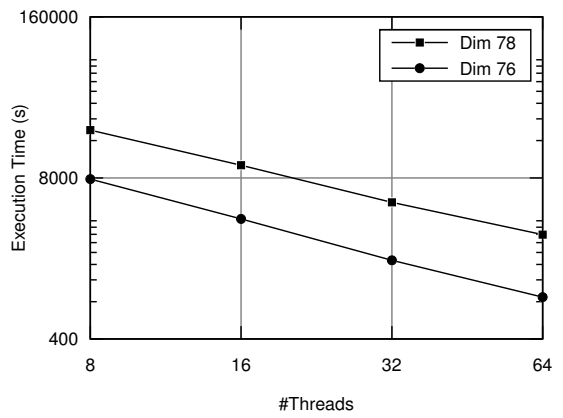

Figure 5. Scalability of our implementation on a 64-core machine, with 8-64 threads. Results for lattices in dimensions 76 and 78. BKZ's block-size is 32. optl is turned off.

might speedup GaussSieve but shift the computation weight to the Klein's algorithm. Either way, we emphasize that (1) a more scalable and efficient kernel of Klein's algorithm must be developed and (2) the proposed implementation of the GaussSieve kernel can be seen as a highly efficient and scalable building block in future implementations.

Figure 5 shows the execution times of our implementation on the 64-core machine, for 8, 16, 32 and 64 threads. This corresponds to the use of one, two, four and eight CPU-chips, respectively. As we are primarily interested in the scalability of our GaussSieve kernel, opt 1 was deactivated in these tests. The version of icpc on this machine is 14.0.2 and the code was also compiled with -02. As the figure shows, our implementation scales almost linearly for up to 64 threads. The speedups and efficiency are shown in Table 5. Our implementation scales linearly for a lattice in dimension 76 and almost linearly for a lattice in dimension 78 . The running times are considerably slower than in the 16-core machine due to the differences in the microarchitectures.

\begin{tabular}{c|c|c|c|c} 
& \multicolumn{2}{|c|}{ Dimension 76 } & \multicolumn{2}{c}{ Dimension 78 } \\
\hline Threads & $\mathrm{S}$ & $\mathrm{E}$ & $\mathrm{S}$ & $\mathrm{E}$ \\
\hline 8 & $7.08 \mathrm{x}$ & $89 \%$ & $7.74 \mathrm{x}$ & $97 \%$ \\
16 & $14.84 \mathrm{x}$ & $93 \%$ & $14.86 \mathrm{x}$ & $93 \%$ \\
32 & $32.02 \mathrm{x}$ & $100 \%$ & 29.65 & $93 \%$ \\
64 & $63.64 \mathrm{x}$ & $99 \%$ & $53.96 \mathrm{x}$ & $84 \%$ \\
\hline
\end{tabular}

Table 5. Speedup (S) and Efficiency (E) of our implementation on the 64-core machine. BKZ's block-size is 32. opt1 is turned off.

\subsection{Comparison of parallel performance}

This section compares the performance of our implementation with the parallel GaussSieve implementations described in [12] and [6], recapped in Section 3. For the sake of simplicity, we refer to these as Milde2011 and Ishiguro2013, respectively. The trials were conducted on the 16-core machine, described in Section 5.

\subsubsection{Comparison with Milde2011}

We ran both implementations with 1-32 threads on a random lattice in dimension 70. Solving lattices in higher dimensions is impractical for less than 32 threads. In this comparison, the lattice was BKZ-reduced, with block-size 32, and we deactivated optl in our implementation, since it degrades the scalability of the GaussReduce kernel, as mentioned in the previous section.

As shown by Figure 6, not only the single-core performance of our implementation is faster than Milde2011, by a factor bigger 


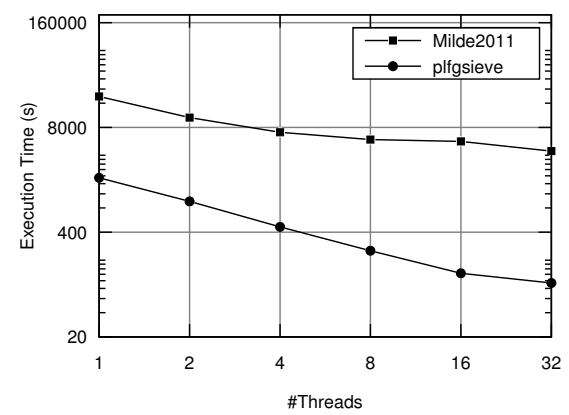

Figure 6. Scalability of our implementation and Milde2011, for 132 threads on the 16-core machine (with SMT). Results for a lattice in dimension 70. BKZ's block-size is 32 . opt1 is turned off.

than 10x, but it also scales much better. In particular, our implementation achieves efficiency levels of $98 \%, 102.25 \%, 100.75 \%, 96 \%$ and $63.28 \%$ (the latter with SMT), whereas Milde2011 achieves only $92 \%, 69.56 \%, 42.75 \%, 22.62 \%$ and $14.92 \%$ (the latter with SMT) for 2, 4, 8, 16 and 32 threads, respectively.

\subsubsection{Comparison with Ishiguro2013}

The Ishiguro2013 implementation is not disclosed, and several implementation details, such as those discussed in Section 3.2, are omitted, thus making a re-implementation impossible. Nevertheless, we can still compare our results with the execution times reported in the paper, since we use the same CPU-chip model. We also replicated the test environment: we ran our implementation with 32 threads, the execution time of the lattice pre-reduction (BKZ with block-size 30) was not measured.

Using 32 threads and $r=8.192$, the authors reported an execution time of 0.9 hours, i.e., 54 minutes or 3240 seconds, for the execution on a random lattice (seed 0) from the SVP-challenge, in dimension 80 (see Section 5.3, Table 2). The execution times for both a random and an ideal lattice are exactly the same, which is surprising, considering that substantial speedups (e.g. $>50 \mathrm{x}$ ) are possible to be achieved for GaussSieve on ideal lattices [14].

Despite of this, our implementation solves the very same lattice in 2896 seconds, i.e. less than 48 and a half minutes (or $\approx 0.8$ hours), which represents an improvement of nearly $12 \%$. In fact, running the Ishiguro2013 implementation with an optimal value for $r$ would still require not less than 45 minutes, i.e. 2700 seconds (see Section 5.1, Figure 3(a)). This is equivalent to a more relaxed stopping criterion on our implementation, since $r$ directly influences the number of iterations required for convergence. In particular, one can compare this result to the most relaxed stopping criterion on our implementation, which is to set a target norm $t n$ as in [15], that permits the algorithm to stop as soon as a vector with norm smaller or equal to $t h$ is found. In this case, with the same 32 threads and BKZ's block-size 30, our implementation runs in 1788 seconds, which represents an improvement factor of more than $1.5 x$.

The authors do not present or comment on the impact of BKZ's block-size on the performance of the GaussSieve, and therefore we assume that 30 is the optimal choice for this parameter on their implementation. On the contrary, we did assess the impact that different block-sizes in BKZ have on the performance of our implementation, as shown in Section 5.2.2. In particular, for BKZ with block-size 34, our implementation solves the SVP on the aforementioned lattice in $\approx 2328$ and $\approx 1591$ seconds, respectively with and without a target norm set. These numbers mean that our implementation is faster by a factor between $\approx 1.39 \mathrm{x}$ and $\approx 1.7 \mathrm{x}$.

\section{Conclusions and Outlook}

This paper proposes a parallel implementation of GaussSieve, an important heuristic that solves the SVP. We show that, by slightly relaxing the properties of GaussSieve, it is possible to achieve almost linear and linear speedups up to 64 cores, depending on the tested scenario. The core idea of the proposed implementation is a lock-free list that holds the vectors in the system, combined with hand-vectorized and hand-optimized code.

In comparison to the previously proposed parallel implementations of GaussSieve, our implementation performs and scales much better than Milde2011, and outperforms Ishiguro2013, by factors of between nearly $1.12 \mathrm{x}$ and $1.50 \mathrm{x}$, for lattices that are BKZ-reduced with block-size 32 , and between nearly $1.39 \mathrm{x}$ and $1.70 \mathrm{x}$, for lattices that are BKZ-reduced with block-size 34.

In the future, we plan to adapt our algorithm to work on ideal lattices, and implement it on CPU+GPU frameworks (e.g. [9]). In this version, we plan to integrate the improvements proposed in [3]. We also plan to implement a parallel version of BKZ on GPUs.

\section{Acknowledgements}

We thank M. Schneider for providing us the implementation showed in [12], and Ö. Dagdelen, L. Santos, T. Laarhoven and F. Correia for insightful discussions. This work was partially supported by the German Science Foundation through SFB 1119 (CROSSING).

\section{References}

[1] M. Ajtai. The Shortest Vector Problem in L2 is NP-hard for Randomized Reductions (Extended Abstract). In Proceedings of the Thirtieth Annual ACM Symposium on Theory of Computing, STOC '98, pages 10-19, New York, NY, USA, 1998. ACM.

[2] M. Ajtai et al.. A Sieve Algorithm for the Shortest Lattice Vector Problem. In Proceedings of the Thirty-third Annual ACM Symposium on Theory of Computing, STOC '01, pages 601-610, New York, NY, USA, 2001. ACM.

[3] R. Fitzpatrick et al. Tuning GaussSieve for Speed. In Third International Conference on Cryptology and Information Security in Latin America (Latincrypt), Florianopolis, Brazil, September 2014.

[4] N. Gama, P. Nguyen, and O. Regev. Lattice Enumeration Using Extreme Pruning. In H. Gilbert, editor, Advances in Cryptology - EUROCRYPT 2010, volume 6110 of Lecture Notes in Computer Science, pages 257-278. Springer Berlin Heidelberg, 2010.

[5] T. L. Harris. A Pragmatic Implementation of Non-blocking LinkedLists. In Proceedings of the 15th International Conference on Distributed Computing, DISC '01, pages 300-314, London, UK, UK, 2001. Springer-Verlag.

[6] T. Ishiguro et al.. Parallel Gauss Sieve Algorithm : Solving the SVP in the Ideal Lattice of 128-dimensions. Cryptology ePrint Archive, Report 2013/388, 2013.

[7] P. Klein. Finding the Closest Lattice Vector when It's Unusually Close. In Proceedings of the Eleventh Annual ACM-SIAM Symposium on Discrete Algorithms, SODA '00, pages 937-941, Philadelphia, PA, USA, 2000. Society for Industrial and Applied Mathematics.

[8] T. Laarhoven et al.. Solving Hard Lattice Problems and the Security of Lattice-Based Cryptosystems. Cryptology ePrint Archive, Report 2012/533, 2012.

[9] A. Mariano et al.. A (ir)regularity-aware task scheduler for heterogeneous platforms. In Proceedings of the Second International Conference on High Performance Computing, HPC-UA'12, pages 45-56, Kiev, Ukraine, October, 8-10 2012.

[10] A. Mariano et al.. A comprehensive empirical comparison of parallel ListSieve and GaussSieve. In To appear in APCI\&E'14 - Workshop on Applications of Parallel Computation in Industry and Engineering, Porto, Portugal, August 2014. 
[11] D. Micciancio and P. Voulgaris. Faster exponential time algorithms for the shortest vector problem. In Proceedings of the Twenty-First Annual ACM-SIAM Symposium on Discrete Algorithms, SODA '10, pages 1468-1480, Philadelphia, PA, USA, 2010. SIAM2.

[12] B. Milde and M. Schneider. A parallel implementation of GaussSieve for the shortest vector problem in lattices. In Proceedings of the 11th International Conference on Parallel computing technologies, PaCT'11, pages 452-458, Berlin, Heidelberg, 2011. Springer-Verlag.

[13] P. Q. Nguyen and et al. Sieve algorithms for the shortest vector problem are practical. J. of Mathematical Cryptology, 2(2), 2008.

[14] M. Schneider. Analysis of Gauss-Sieve for Solving the Shortest Vector Problem in Lattices. In WALCOM: Algorithms and Computation, volume 6552 of Lecture Notes in Computer Science, pages 89-97. Springer Berlin Heidelberg, 2011.

[15] M. Schneider. Sieving for Shortest Vectors in Ideal Lattices. In Progress in Cryptology AFRICACRYPT 2013, volume 7918 of Lecture Notes in Computer Science, pages 375-391. Springer Berlin Heidelberg, 2013.

[16] P. Xavier et al.. Solving the shortest lattice vector problem in time $2^{2} .465 n$. Cryptology ePrint Archive, Report 2009/605, 2009.

[17] W. Xiaoyun et al.. Improved Nguyen-Vidick heuristic sieve algorithm for shortest vector problem. In In Proceedings of ASIACCS 11, pages 1-9. ACM, 2011.

[18] F. Zhang et al.. A Three-Level Sieve Algorithm for the Shortest Vector Problem. In SAC 2013 - 20th International Conference on Selected Areas in Cryptography, Burnaby, Canada, August 2013. 